\title{
Long-term clinical outcomes of bilateral GPi deep brain stimulation in advanced Parkinson's disease: 5 years and beyond
}

\author{
Takashi Tsuboi, MD, PhD, ${ }^{1,2}$ Janine Lemos Melo Lobo Jofili Lopes, MD, ${ }^{1}$ Kathryn Moore, MD, ${ }^{1}$ \\ Bhavana Patel, DO, ' Joseph Legacy, MD, ${ }^{1}$ Adrianna M. Ratajska, BS, 1,3 Dawn Bowers, PhD, 1,3 \\ Robert S. Eisinger, PhD, ${ }^{1}$ Leonardo Almeida, MD, ${ }^{1}$ Kelly D. Foote, MD, ${ }^{4}$ Michael S. Okun, MD, ${ }^{1}$ and \\ Adolfo Ramirez-Zamora, MD ${ }^{1}$
}

\begin{abstract}
1Department of Neurology, Norman Fixel Institute for Neurological Diseases, and Departments of ${ }^{3}$ Clinical and Health Psychology and ${ }^{4}$ Neurosurgery, University of Florida, Gainesville, Florida; and ${ }^{2}$ Department of Neurology, Nagoya University Graduate School of Medicine, Nagoya, Aichi, Japan
\end{abstract}

OBJECTIVE Few studies have reported long-term outcomes of globus pallidus internus (GPi) deep brain stimulation (DBS) in Parkinson's disease (PD). The authors aimed to investigate long-term outcomes of bilateral GPi DBS for 5 years and beyond for PD patients.

METHODS The authors retrospectively analyzed the clinical outcomes in 65 PD patients treated with bilateral GPi DBS at a single center. The outcome measures of motor symptoms and health-related quality of life (HRQoL) included the Unified Parkinson's Disease Rating Scale (UPDRS) and the Parkinson's Disease Questionnaire (PDQ-39). Scores at baseline were compared with those at 1, 3, 5, and 6-8 years after implantation using Wilcoxon signed-rank tests with $\alpha$ correction. RESULTS GPi DBS significantly improved the off-medication UPDRS III total scores, UPDRS IV, and dyskinesia score at 1 year when compared with baseline (all $p<0.001$ ). The off- and on-medication tremor scores, UPDRS IV, and dyskinesia scores showed moderate and sustained improvement (the ranges of the mean percentage improvement at each time point were $61 \%-75 \%, 30 \%-80 \%, 29 \%-40 \%$, and $40 \%-65 \%$, respectively) despite lacking statistical significance at long-term follow-up with diminishing sample sizes. The off-medication UPDRS III total scores did not show significant improvement at 5 years or later, primarily because of worsening in rigidity, akinesia, speech, gait, and postural stability scores. The on-medication UPDRS III total scores also worsened over time, with a significant worsening at 6-8 years when compared with baseline $(p=0.008)$. The HRQoL analyses based on the PDQ-39 revealed significant improvement in the activities of daily living and discomfort domains at 1 year ( $p=0.003$ and 0.006 , respectively); however, all the domains showed gradual worsening at the later time points without reaching statistical significance. At 3 years, the communication domain showed significant worsening compared with baseline scores $(p=0.002)$.

CONCLUSIONS GPi DBS in PD patients in this single-center cohort was associated with sustained long-term benefits in the off- and on-medication tremor score and motor complications. HRQoL and the cardinal motor symptoms other than tremor may worsen gradually in the long term. When counseling patients, it is important to recognize that benefits in tremor and dyskinesia are expected to be most persistent following bilateral GPi DBS implantation.

https://thejns.org/doi/abs/10.3171/2020.6.JNS20617

KEYWORDS Parkinson's disease; deep brain stimulation; globus pallidus internus; quality of life; functional neurosurgery

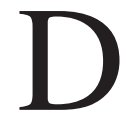
EEP brain stimulation (DBS) targeting the globus pallidus internus (GPi) and subthalamic nucleus (STN) provides similar benefits for motor symptoms and health-related quality of life (HRQoL) in Parkinson's disease (PD) patients with medically refractory motor fluctuations. ${ }^{1}$ In large randomized trials, bilateral GPi DBS significantly improved activities of daily living (ADLs) and dyskinesia as well as motor symptoms in the off-medication state when measured at different follow-up intervals following DBS implantation (1-3 years). ${ }^{2,3}$ Addi-

ABBREVIATIONS ADL = activity of daily living; AE = adverse events; BDI-II = Beck Depression Inventory-II; DBS = deep brain stimulation; DRS-2 = Dementia Rating Scale-2; GPi = globus pallidus internus; HRQoL = health-related quality of life; LEDD = levodopa equivalent daily dose; PD = Parkinson's disease; PDQ-39 = Parkinson's Disease Questionnaire; SAS = Starkstein Apathy Scale; STN = subthalamic nucleus; UPDRS = Unified Parkinson's Disease Rating Scale.

SUBMITTED February 27, 2020. ACCEPTED June 5, 2020.

INCLUDE WHEN CITING Published online October 9, 2020; DOI: 10.3171/2020.6.JNS20617. 
tionally, one of these studies reported sustained improvement in the ADL, stigma, and body discomfort domains based on the Parkinson's Disease Questionnaire (PDQ-39) at 3 years following bilateral GPi DBS implantation. ${ }^{2}$

To the best of our knowledge, only 3 studies have reported at least 5-year motor outcomes following GPi DBS. These studies total only 30 patients combined. ${ }^{4-6}$ All the studies reported the ADLs and motor outcomes based on the Unified Parkinson's Disease Rating Scale (UPDRS) II and III, respectively. Volkmann et al. reported 5-year GPi DBS outcomes in 6 PD patients. ${ }^{4}$ At 5 years, significant improvement in the off-medication UPDRS II and III compared with baseline were lost; however, a significant reduction of dyskinesia based on the dyskinesia rating scale was maintained (64\%). ${ }^{4}$ Moro et al. reported the GPi DBS outcomes in 16 patients at 5-6 years, and there was persistent improvement in the off-medication UPDRS II and III compared with baseline (36\% and 37\%, respectively). ${ }^{5}$ Also, a stable reduction of dyskinesia based on the UPDRS IV was observed (75\% reduction compared with baseline). ${ }^{5}$ Lachenmayer et al. showed that GPi DBS significantly improved the off-medication UPDRS III scores by $22 \%$ at 5.5 years without significant improvement in the off-medication UPDRS II $(\mathrm{n}=8){ }^{6}$ Also, UPDRS IV and its dyskinesia and motor fluctuation subscores remained significantly improved by $41 \%, 49 \%$, and $38 \%$, respectively. ${ }^{6}$ None of these studies observed significant improvement in the on-medication UPDRS III at 5 years. The impacts on cardinal motor symptoms were inconsistent among these studies, and significant improvement was observed in the off-medication tremor and/or rigidity subscores at 5 years. ${ }^{4-6}$ Variable outcomes in these studies may result from the differences in patient clinical characteristics, management, and unique disease progression. Additionally, HRQoL outcomes were not reported in these 3 studies.

In the present study, we aimed to determine the longterm ( $\geq 5$ years) motor and HRQoL outcomes of GPi DBS for PD patients based on the UPDRS and PDQ-39.

\section{Methods \\ Inclusion Criteria}

In this retrospective study, we collected patient data from the IRB-approved database at the University of Florida, Gainesville. The inclusion criteria were as follows: 1) diagnosis of PD based on the UK Parkinson's Disease Society brain bank criteria; ${ }^{7}$ 2) bilateral GPi DBS performed at the University of Florida, Gainesville, between January 2007 and August 2018; 3) no prior history of stereotactic brain surgery; and 4) preoperative clinical assessment data with postoperative follow-up data for 1 year or beyond. DBS implantations were typically staged as this was the standard of care for the University of Florida. Individuals who underwent staged (nonsimultaneous) bilateral GPi DBS surgeries with an interval of 9 months or more were excluded to minimize the impact of disease progression.

\section{Standard Perioperative Procedures}

Our multidisciplinary team evaluated all patients to determine a favorable risk-benefit ratio before DBS im- plantation. We also carefully consider patients' symptom profiles (e.g., existence and severity of dyskinesia, dystonia, axial symptoms, or cognitive and mood problems) when offering GPi or STN DBS. ${ }^{1}$ In general, patients with severe dyskinesia, balance/gait problems, or cognitive concerns were more likely to undergo GPi DBS instead of STN DBS. Surgical procedures were performed as previously described. ${ }^{8}$ Briefly, DBS leads (model 3387; Medtronic) were implanted under local anesthesia using microelectrode recordings and macrostimulation testing. The latest available pulse generators (Activa PC/SC, Soletra, or Kinetra; Medtronic) were implanted. A team comprising neurologists and nurses specializing in movement disorders and DBS evaluated the patients monthly for the first 6 months to optimize stimulation settings and medications. This procedure was followed by visits every 3 months up to 1 year and annual visits thereafter. The patients had additional visits if clinically necessary. Assessments from clinic visits through November 2019 were analyzed.

\section{Outcome Measures and Postoperative Time Points}

Clinical assessments included the UPDRS and PDQ39. ${ }^{9}$ UPDRS III scores were completed in the off-medication state, defined as a cessation of dopaminergic medications 12 hours before their visit. Subsequently, patients underwent on-medication motor assessments, approximately 60 minutes after taking their medications. The DBS devices remained "on" at postoperative evaluations. We extracted tremor total (items 20 and 21), resting tremor (item 20), postural/action tremor (item 21), rigidity (item 22), akinesia (items 23-26 and 31), axial total (items 27 30), speech (item 18), gait (item 29), and postural stability (item 30 ) scores from the UPDRS III and dyskinesia (items 32-34) scores from the UPDRS IV for further analyses. Levodopa equivalent daily dose (LEDD) was calculated as previously described. ${ }^{10}$ As part of the preoperative evaluation, cognitive function, depressive symptoms, and apathy were assessed using the Dementia Rating Scale-2 (DRS-2), the Beck Depression Inventory-II (BDI-II), and the Starkstein Apathy Scale (SAS), respectively. ${ }^{11-13}$ The postoperative evaluations were divided into 4 time points (1, 3, 5, and 6-8 years). For 1-, 3-, and 5-year time points, we allowed for \pm 0.5 -year intervals to capture all of the appropriate follow-up visits. For the patients who were lost to follow-up at 3 years or later, all the available baseline and postoperative data were included in the analysis.

\section{Statistical Analysis}

Statistical analysis was performed using IBM SPSS statistical software (version 25, IBM Corp.). The normal distribution of data was tested using the Shapiro-Wilk test. We compared baseline characteristics between the groups using independent t-tests, Mann-Whitney U-tests, or chisquare tests as appropriate. The statistical significance was set to a threshold of $p<0.05$. The outcome measures at each follow-up were compared with the baseline status using Wilcoxon signed-rank tests with Holm-Bonferroni correction for multiple comparisons, which adjusts $\mathrm{p}$ values for each hypothesis with a range of significance 
TABLE 1. Baseline clinical characteristics of the patients included and those excluded from the analysis

\begin{tabular}{lccc}
\hline \multicolumn{1}{c}{ Variable } & $\begin{array}{c}\text { Patients } \\
\text { Included }\end{array}$ & $\begin{array}{c}\text { Patients } \\
\text { Excluded }\end{array}$ & $\begin{array}{c}p \\
\text { Value }\end{array}$ \\
\hline No. of patients & 65 & 20 & \\
\hline Men (\%) & $39(60 \%)$ & $16(80 \%)$ & 0.102 \\
\hline Age at DBS implantation, yrs & $62.6(9.2)$ & $61.8(6.9)$ & 0.432 \\
\hline Disease duration before DBS, yrs & $11.9(5.4)$ & $12.9(7.9)$ & 0.855 \\
\hline UPDRS I & $2.8(2.0)$ & $2.6(1.8)$ & 0.890 \\
\hline UPDRS II & $17.5(6.1)$ & $17.3(6.2)$ & 0.896 \\
\hline UPDRS III, off-med & $42.8(10.1)$ & $38.3(11.4)$ & 0.162 \\
\hline UPDRS III, on-med & $24.3(8.7)$ & $24.2(11.0)$ & 0.805 \\
\hline UPDRS IV & $7.5(3.2)$ & $8.5(3.0)$ & 0.229 \\
\hline Hoehn \& Yahr stage & $2.9(0.7)$ & $2.7(0.9)$ & 0.149 \\
\hline DRS-2 & $136.5(5.0)$ & $134.3(7.7)$ & 0.521 \\
\hline BDI-II & $9.1(6.9)$ & $10.2(7.1)$ & 0.546 \\
\hline SAS & $10.7(6.3)$ & $10.2(6.0)$ & 0.803 \\
\hline LEDD & $1273(553)$ & $1372(682)$ & 0.518 \\
\hline
\end{tabular}

Med $=$ medication .

Data are presented as mean (SD) unless otherwise indicated. The clinical characteristics of patients who completed regular visits and those who were lost to follow-up were compared using independent t-tests, Mann-Whitney U-tests, or chi-square tests as appropriate.

thresholds $(0.0125-0.05) .^{14}$ The postoperative UPDRS III scores (off- and on-medication states) were compared with the baseline UPDRS III scores (off- and on-medication states).

\section{Results}

\section{Characteristics of the Cohort}

From the 85 PD patients who underwent bilateral GPi DBS surgeries at our center between January 2007 and August 2018, we excluded 20 patients who underwent staged surgeries with an interval longer than 9 months. There were no significant differences in the baseline clinical characteristics (demographics, UPDRS, Hoehn and Yahr stage, DRS-2, BDI-II, SAS, and LEDD) between the patients included and those excluded from the analysis (Table 1). Consequently, the cohort comprised 65 patients with a mean age at DBS implantation of $62.6 \pm 9.2$ years and a disease duration before DBS of $11.9 \pm 5.4$ years. Not all patients had completed long-term follow-up assessments at the time of our analysis (based on individual dates of DBS implantation). Additionally, 12 of the 65 patients were lost to follow-up (6 patients at the 3 -year time point, 4 between the 3- and 5-year time points, and 2 between the 5- and 6- to 8-year time points) for the following reasons: transferring care to a different DBS center $(\mathrm{n}=$ $6)$, moving into a long-term care facility with an inability to return to the clinic for follow-ups $(n=2)$, death $(n=3)$, and unclear reasons $(\mathrm{n}=1)$. When comparing the clinical characteristics of the patients who completed regular visits to date and those who were lost to follow-up (Table 2), we did not find any significant differences except for sex $(\mathrm{p}$ $=0.012$ ). There were some missing data on each variable,
TABLE 2. Baseline clinical characteristics of the patients who completed regular visits and those lost to follow-up

\begin{tabular}{lccc}
\hline \multicolumn{1}{c}{ Variable } & $\begin{array}{c}\text { Patients Who } \\
\text { Completed } \\
\text { Regular Visits }\end{array}$ & $\begin{array}{c}\text { Patients } \\
\text { Lost to } \\
\text { Follow-Up }\end{array}$ & $\begin{array}{c}\text { p } \\
\text { Value }\end{array}$ \\
\hline No. of patients & 53 & 12 & \\
\hline Men (\%) & $28(53 \%)$ & $11(92 \%)$ & $0.012^{*}$ \\
\hline Age at DBS implantation, yrs & $62.5(9.0)$ & $62.9(10.5)$ & 0.990 \\
\hline Disease duration before DBS, yrs & $12.4(5.4)$ & $9.8(5.4)$ & 0.117 \\
\hline UPDRS I & $2.8(2.1)$ & $2.4(1.7)$ & 0.607 \\
\hline UPDRS II & $17.6(6.2)$ & $17.0(5.9)$ & 0.660 \\
\hline UPDRS III, off-med & $43.1(10.3)$ & $41.4(9.6)$ & 0.812 \\
\hline UPDRS III, on-med & $24.5(8.9)$ & $23.5(8.1)$ & 0.970 \\
\hline UPDRS IV & $7.4(3.2)$ & $7.8(3.1)$ & 0.688 \\
\hline Hoehn \& Yahr stage & $2.9(0.7)$ & $2.8(0.9)$ & 0.510 \\
\hline DRS-2 & $136.9(4.2)$ & $134.9(7.5)$ & 0.624 \\
\hline BDI-II & $9.3(6.9)$ & $8.1(7.1)$ & 0.537 \\
\hline SAS & $10.7(6.0)$ & $10.8(7.9)$ & 0.662 \\
\hline LEDD & $1227(534)$ & $1476(621)$ & 0.185 \\
\hline
\end{tabular}

Data are presented as mean (SD) unless otherwise indicated. The clinical characteristics of patients who completed regular visits and those who were lost to follow-up were compared using independent t-tests, Mann-Whitney U-tests, or chi-square tests as appropriate.

* Statistically significant $(p<0.05)$.

and the number of patients available for each analysis is shown in Table 3.

\section{UPDRS Outcomes After DBS}

UPDRS IV, dyskinesia score, and Hoehn and Yahr stage significantly improved at 1 year after DBS implantation $(\mathrm{p}<0.001, \mathrm{p}<0.001$, and $\mathrm{p}=0.009$, respectively; Table 3, Fig. 1). UPDRS IV showed comparable magnitudes of improvement at the short- and long-term assessments (mean percentage improvement: $40 \%$ at 1 year, $33 \%$ at 3 years, $30 \%$ at 5 years, and $29 \%$ at $6-8$ years), with a statistically significant benefit for up to 3 years (all $p<0.01$; Fig. 1). Percentage improvement in UPDRS IV $\geq 50 \%$ was observed in $33 \%$ and $29 \%$ of the individual patients at 5 and 6-8 years, respectively (Table 4). In addition, we observed relatively stable long-term outcomes for the dyskinesia scores (mean percentage improvement: 65\% at 1 year, $40 \%$ at 3 years, $50 \%$ at 5 years, and $50 \%$ at $6-8$ years), with statistical significance only at 1 year $(\mathrm{p}<0.001$; Fig. 1). Percentage improvement in dyskinesia scores $\geq 50 \%$ was observed in $90 \%$ and $80 \%$ of the patients at 5 and $6-8$ years, respectively (Table 4). UPDRS II showed a trend for improvement at 1 year without statistical significance after $\alpha$ correction $(\mathrm{p}=0.042$; Table 3$)$.

GPi DBS significantly improved the off-medication UPDRS III total scores with a significant reduction in tremor total, resting tremor, postural/action tremor, rigidity, akinesia, axial symptoms total, and postural stability scores at 1 year when compared with baseline scores (all $\mathrm{p}<0.05$; Table 3, Fig. 1). The off-medication UPDRS III total scores did not show significant differences at 5 years or later primarily because of worsening in rigidity, aki- 


\begin{tabular}{|c|c|c|c|c|c|c|c|c|c|c|c|c|c|c|}
\hline \multirow[b]{2}{*}{ Measure } & \multirow[b]{2}{*}{$\mathrm{N}$} & \multirow[b]{2}{*}{$B L$} & \multirow[b]{2}{*}{$\mathrm{N}$} & \multirow[b]{2}{*}{$1 \mathrm{Yr}$} & \multirow[b]{2}{*}{$\mathrm{N}$} & \multirow[b]{2}{*}{3 Yrs } & \multirow[b]{2}{*}{$\mathrm{N}$} & \multirow[b]{2}{*}{5 Yrs } & \multirow[b]{2}{*}{$\mathrm{N}$} & \multirow[b]{2}{*}{$6-8$ Yrs } & \multicolumn{4}{|c|}{$p$ Value } \\
\hline & & & & & & & & & & & $\begin{array}{c}\text { BL vs } \\
1 Y r\end{array}$ & $\begin{array}{l}\text { BL vs } \\
3 \text { Yrs }\end{array}$ & $\begin{array}{l}\text { BL vs } \\
5 \text { Yrs }\end{array}$ & $\begin{array}{l}\text { BL vs } \\
6-8 \text { Yrs }\end{array}$ \\
\hline UPDRS II & 65 & $17.5(6.1)$ & 61 & $15.7(7.2)$ & 33 & $18.7(7.2)$ & 16 & $19.8(8.7)$ & 7 & $24.6(8.0)$ & $0.042^{*}$ & 0.589 & 0.909 & 0.109 \\
\hline UPDRS IV & 56 & $7.5(3.2)$ & 57 & $4.4(2.3)$ & 32 & $4.5(2.4)$ & 13 & $4.5(2.3)$ & 7 & $5.0(2.2)$ & $<0.001 \uparrow$ & $0.001 \dagger$ & $0.047 \dagger$ & 0.250 \\
\hline $\begin{array}{l}\text { Dyskinesia total } \\
\text { (items 32-34) }\end{array}$ & 59 & $2.3(2.3)$ & 58 & $0.8(1.1)$ & 32 & $0.9(1.2)$ & 14 & $0.8(1.2)$ & 7 & $0.6(0.5)$ & $<0.001 \dagger$ & 0.070 & 0.071 & 0.313 \\
\hline Hoehn \& Yahr stage & 59 & $2.9(0.7)$ & 61 & $2.6(0.8)$ & 32 & $2.8(0.9)$ & 15 & $3.5(1.2)$ & 7 & $3.4(1.0)$ & $0.009 \dagger$ & 0.836 & 0.310 & $>0.999$ \\
\hline LEDD & 65 & $1273(555)$ & 65 & $1356(660)$ & 35 & 1335 (703) & 16 & $1226(710)$ & 8 & $1383(722)$ & 0.288 & 0.665 & 0.207 & 0.313 \\
\hline \multicolumn{15}{|l|}{ UPDRS III, off-med } \\
\hline Total score & 65 & $42.8(10.1)$ & 49 & $30.2(8.7)$ & 17 & $36.6(9.6)$ & 9 & $38.8(7.3)$ & 3 & $44.3(4.0)$ & $<0.001 \dagger$ & $0.012 \dagger$ & 0.102 & 0.500 \\
\hline $\begin{array}{l}\text { Tremor total (items } \\
20 \& 21 \text { ) }\end{array}$ & 65 & $6.6(5.1)$ & 49 & $2.5(3.3)$ & 17 & $2.4(2.7)$ & 9 & $1.4(1.4)$ & 3 & $1.7(2.1)$ & $<0.001 \dagger$ & $0.001 \dagger$ & $0.023 \dagger$ & 0.500 \\
\hline Rigidity (item 22) & 65 & $9.0(3.0)$ & 49 & $6.3(3.1)$ & 17 & $7.0(3.2)$ & 9 & $7.9(3.2)$ & 3 & $8.7(1.5)$ & $<0.001 \uparrow$ & $0.023^{*}$ & 0.125 & 0.750 \\
\hline $\begin{array}{l}\text { Akinesia total } \\
\text { (items 23-26, 31) }\end{array}$ & 65 & $18.0(4.8)$ & 49 & $13.7(4.3)$ & 17 & $16.4(4.8)$ & 9 & $16.3(2.5)$ & 3 & $17.7(4.0)$ & $<0.001 \dagger$ & $0.026^{*}$ & 0.094 & 0.750 \\
\hline $\begin{array}{l}\text { Axial symptoms } \\
\text { total (items 27-30) }\end{array}$ & 65 & $5.9(2.7)$ & 49 & $4.6(2.8)$ & 17 & $7.1(3.2)$ & 9 & $9.0(2.9)$ & 3 & $11.0(2.6)$ & $<0.001 \dagger$ & 0.876 & 0.383 & 0.500 \\
\hline Speech (item 18) & 65 & $1.2(0.7)$ & 49 & $1.5(0.6)$ & 17 & $1.9(0.6)$ & 9 & $2.1(0.3)$ & 3 & $2.7(0.6)$ & 0.690 & 0.398 & 0.250 & 0.250 \\
\hline Gait (item 29) & 65 & $1.0(0.8)$ & 49 & $1.5(0.9)$ & 17 & $2.3(0.8)$ & 9 & $2.7(0.7)$ & 3 & $3.3(0.6)$ & $0.025^{*}$ & 0.641 & 0.063 & 0.500 \\
\hline $\begin{array}{l}\text { Postural stability } \\
\text { (item 30) }\end{array}$ & 65 & $0.7(0.8)$ & 49 & $1.0(1.0)$ & 17 & $1.8(1.2)$ & 9 & $2.2(1.0)$ & 3 & $3.0(1.0)$ & $0.002 \dagger$ & 0.772 & 0.438 & 0.500 \\
\hline Rigidity (item 22) & 65 & $5.0(2.9)$ & 65 & $4.4(2.6)$ & 35 & $4.8(2.5)$ & 16 & $6.0(1.8)$ & 8 & $7.8(2.5)$ & 0.085 & 0.746 & $>0.999$ & $0.016^{*}$ \\
\hline $\begin{array}{l}\text { Akinesia total } \\
\text { (items 23-26, 31) }\end{array}$ & 65 & $10.9(4.1)$ & 65 & $10.4(4.6)$ & 35 & $12.5(4.3)$ & 16 & $14.6(5.7)$ & 8 & $15.9(6.5)$ & 0.661 & 0.089 & 0.212 & $0.031^{*}$ \\
\hline $\begin{array}{l}\text { Axial symptoms } \\
\text { total (items } 27-30 \text { ) }\end{array}$ & 65 & $3.2(2.3)$ & 65 & $3.7(2.7)$ & 35 & $5.6(3.1)$ & 16 & $7.4(4.1)$ & 8 & $7.1(3.0)$ & 0.103 & $0.002 \dagger$ & $0.021 \dagger$ & $0.016 \dagger$ \\
\hline Speech (item 18) & 65 & $1.3(0.6)$ & 65 & $1.3(0.6)$ & 35 & $1.7(0.7)$ & 16 & $2.1(0.4)$ & 8 & $2.5(0.9)$ & 0.269 & $0.006 \dagger$ & $0.002 \dagger$ & 0.094 \\
\hline Gait (item 29) & 65 & $1.1(0.9)$ & 65 & $1.1(0.9)$ & 35 & $1.6(0.9)$ & 16 & $2.1(0.9)$ & 8 & $2.4(1.1)$ & 0.443 & $0.010 \dagger$ & $0.009 \dagger$ & $0.016 \dagger$ \\
\hline $\begin{array}{l}\text { Postural stability } \\
\text { (item 30) }\end{array}$ & 65 & $0.8(0.9)$ & 65 & $0.8(0.9)$ & 35 & $1.3(1.0)$ & 16 & $1.9(1.3)$ & 8 & $1.5(1.1)$ & 0.243 & $0.023^{*}$ & 0.148 & 0.438 \\
\hline \multicolumn{15}{|l|}{ PDQ-39 } \\
\hline Mobility & 50 & $49.0(27.9)$ & 51 & $39.7(22.7)$ & 27 & $50.4(26.5)$ & 12 & $60.8(24)$ & 7 & $61.4(20.2)$ & 0.064 & 0.837 & 0.864 & 0.813 \\
\hline$A D L$ & 50 & $41.9(25.7)$ & 51 & $29.7(20.5)$ & 27 & $41.5(24.0)$ & 12 & $47.2(30.4)$ & 7 & $52.4(25.1)$ & $0.003 \dagger$ & 0.626 & 0.971 & 0.813 \\
\hline Emotional & 51 & $28.6(22.0)$ & 51 & $22.6(17.6)$ & 27 & $32.5(21.7)$ & 12 & $31.9(17.4)$ & 7 & 36.9 (19.2) & 0.125 & 0.314 & 0.372 & 0.844 \\
\hline Stigma & 51 & $22.7(24.1)$ & 51 & $18.6(18.6)$ & 27 & $20.6(19.8)$ & 12 & $25.0(22.8)$ & 7 & $25.9(15.9)$ & 0.210 & 0.306 & 0.801 & $>0.999$ \\
\hline Social & 49 & $11.4(18.9)$ & 51 & $13.2(16.3)$ & 27 & $22.3(24.7)$ & 12 & $20.1(26.9)$ & 7 & $21.4(20.9)$ & 0.199 & 0.240 & 0.344 & 0.625 \\
\hline Cognition & 51 & $26.2(19.5)$ & 51 & $24.1(17.1)$ & 27 & $35.1(20.7)$ & 12 & $31.3(12.8)$ & 7 & $33.9(24.4)$ & 0.351 & 0.469 & $>0.999$ & 0.375 \\
\hline
\end{tabular}




\begin{tabular}{|c|c|c|c|c|c|c|c|c|c|c|c|c|c|c|}
\hline \multirow[b]{2}{*}{ Measure } & \multirow[b]{2}{*}{$\mathrm{N}$} & \multirow[b]{2}{*}{$\mathrm{BL}$} & \multirow[b]{2}{*}{$\mathrm{N}$} & \multirow[b]{2}{*}{$1 \mathrm{Yr}$} & \multirow[b]{2}{*}{$\mathrm{N}$} & \multirow[b]{2}{*}{3 Yrs } & \multirow[b]{2}{*}{$\mathrm{N}$} & \multirow[b]{2}{*}{5 Yrs } & \multirow[b]{2}{*}{$\mathrm{N}$} & \multirow[b]{2}{*}{$6-8$ Yrs } & \multicolumn{4}{|c|}{$p$ Value } \\
\hline & & & & & & & & & & & $\begin{array}{c}\text { BL vs } \\
1 \mathrm{Yr}\end{array}$ & $\begin{array}{l}\text { BL vs } \\
3 \text { Yrs }\end{array}$ & $\begin{array}{l}\text { BL vs } \\
5 \text { Yrs }\end{array}$ & $\begin{array}{c}\text { BL vs } \\
6-8 \text { Yrs }\end{array}$ \\
\hline Communication & 51 & $24.5(20.4)$ & 51 & $29.6(17.8)$ & 27 & $42.6(28.5)$ & 12 & $38.9(29.4)$ & 7 & $54.8(24.9)$ & 0.078 & $0.005 \dagger$ & 0.211 & 0.344 \\
\hline Discomfort & 51 & $46.1(25.4)$ & 51 & $35.9(24.6)$ & 26 & $45.5(26.5)$ & 12 & $37.5(28.8)$ & 7 & $47.6(22.4)$ & $0.006 \dagger$ & 0.804 & 0.217 & 0.500 \\
\hline
\end{tabular}

$\mathrm{BL}=$ baseline.

Data are presented as mean (SD). Statistical analysis was performed using Wilcoxon signed-rank tests. A p value $<0.05$ was considered significant.

${ }^{*} p$ values that were $<0.05$ but did not reach statistical significance after $\alpha$ correction for multiple comparisons.

$\dagger p$ values that reached statistical significance after $\alpha$ correction for multiple comparisons.

$\ddagger$ The postoperative assessments were performed with DBS "on."

nesia, speech, gait, and postural stability scores. Tremor total scores showed statistically significant improvements up to 5 years (all $\mathrm{p}<0.05)$ and showed comparable mean percentage improvement over time $(61 \%$ at 1 year, $64 \%$ at 3 years, $75 \%$ at 5 years, and $62 \%$ at $6-8$ years; Fig. 1). Percentage improvement in the tremor total scores $\geq 50 \%$ was observed in $78 \%$ and $67 \%$ of the patients at 5 years and 6-8 years, respectively (Table 5 ). Statistically significant improvement in resting tremor and postural/action tremor scores was observed up to 3 years after $\alpha$ correction. In contrast, rigidity, akinesia, and postural stability scores declined. Gait scores showed a trend for improvement at 1 year without statistical significance after $\alpha$ correction ( $\mathrm{p}$ $=0.025$ ).

In the on-medication state, GPi DBS significantly improved tremor total scores as well as resting and postural/ action tremor at 1 year when compared with baseline (all $p$ $<0.001$; Table 3, Fig. 1). Furthermore, the improvement in tremor total scores was sustained in the long term (mean percentage improvement: $57 \%$ at 1 year, $69 \%$ at 3 years, $80 \%$ at 5 years, and $30 \%$ at $6-8$ years) and showed a statistical significance up to 5 years (all $p<0.05$; Fig. 1). A percentage improvement in the tremor total scores $\geq 50 \%$ was observed in $75 \%$ and $40 \%$ of the patients at 5 and 6-8 years, respectively (Table 5). In contrast, UPDRS III total scores worsened over time, along with rigidity, akinesia, speech, gait, and postural stability, and a significant worsening in the UPDRS III total scores was observed at $6-8$ years ( $p=0.008$; Table 3 , Fig. 1$)$. Axial symptom total scores showed significant worsening at 3 years and later (all $\mathrm{p}<0.05$; Fig. 1). Rigidity and akinesia scores showed a trend for worsening at 6-8 years without statistical significance after $\alpha$ correction ( $\mathrm{p}=0.016$ and 0.031 , respectively; Fig. 1).

Percentage improvement in UPDRS IV, dyskinesia, and off- and on-medication tremor scores for the patients who were lost to follow-up is presented in Supplementary Tables 1 and 2. Although we did not apply statistical analysis because of the small numbers of patients lost to follow-up, the results of the whole cohort and the patients lost to follow-up were comparable.

\section{HRQoL Outcomes}

One year after GPi DBS, there was significant improve- ment in the ADL and discomfort domains of the PDQ-39 $(p=0.003$ and 0.006 , respectively; Table 3 ). At 3 years, the communication domain showed significant worsening compared with baseline scores $(\mathrm{p}=0.005)$. All the domains showed gradual worsening at the later time points without a statistical significance.

\section{Medication, Electrode Positions, and DBS Programming Parameters}

At a group level, there were no significant changes in LEDD postoperatively, as presented in Table 3. Before DBS implantation, the patients were treated with levodopa $(100 \%)$ in combination with other antiparkinsonian medications, including dopamine agonists (52\%), amantadine (26\%), monoamine oxidase type B (MAOB) inhibitors $(25 \%)$, and catechol- $O$-methyltransferase (COMT) inhibitors (22\%), as summarized in Supplementary Table 3. During the follow-up, the usage of COMT inhibitors tended to increase; conversely, the usage of dopamine agonists, amantadine, and MAOB inhibitors tended to decrease (statistical analysis not applied).

The anatomical coordinates of the electrode tips relative to the midcommissural points were as follows: -21.7 $\pm 1.5,0.4 \pm 1.7$, and $-5.8 \pm 2.1$ for the left GPi; $21.5 \pm 1.6$, $1.2 \pm 1.5$, and $-5.5 \pm 1.1$ for the right GPi (x [m], lateral to midline; $y[\mathrm{~mm}]$, anterior to the midcommissural point; and $\mathrm{z}[\mathrm{mm}]$, ventral to the intercommissural plane).

The DBS programming parameters at each time point are summarized in Table 6. At 1 year, the medians (ranges) of the DBS programming parameters were as follows: voltage, $2.8 \mathrm{~V}(1.6-4.6 \mathrm{~V})$; pulse width, 90 usec (60-150 $\mu \mathrm{sec})$; and stimulation frequency, $160 \mathrm{~Hz}(135-200 \mathrm{~Hz})$. At a group level, the DBS parameters remained similar during the follow-up period, although different combinations of DBS parameters were empirically tried to maximize potential benefits.

\section{Adverse Events}

Information on the surgery-, device-, and stimulationrelated adverse events (AEs) was collected. The most common AEs were gait/postural disorders $(n=12)$, stimulation-induced dysarthria $(\mathrm{n}=9)$, and lead fractures $(\mathrm{n}$ $=8)$. Other AEs included dyskinesia $(n=4)$, cognitive decline $(n=2)$, stimulation-induced paresthesia $(n=2)$, 
Tsuboi et al.
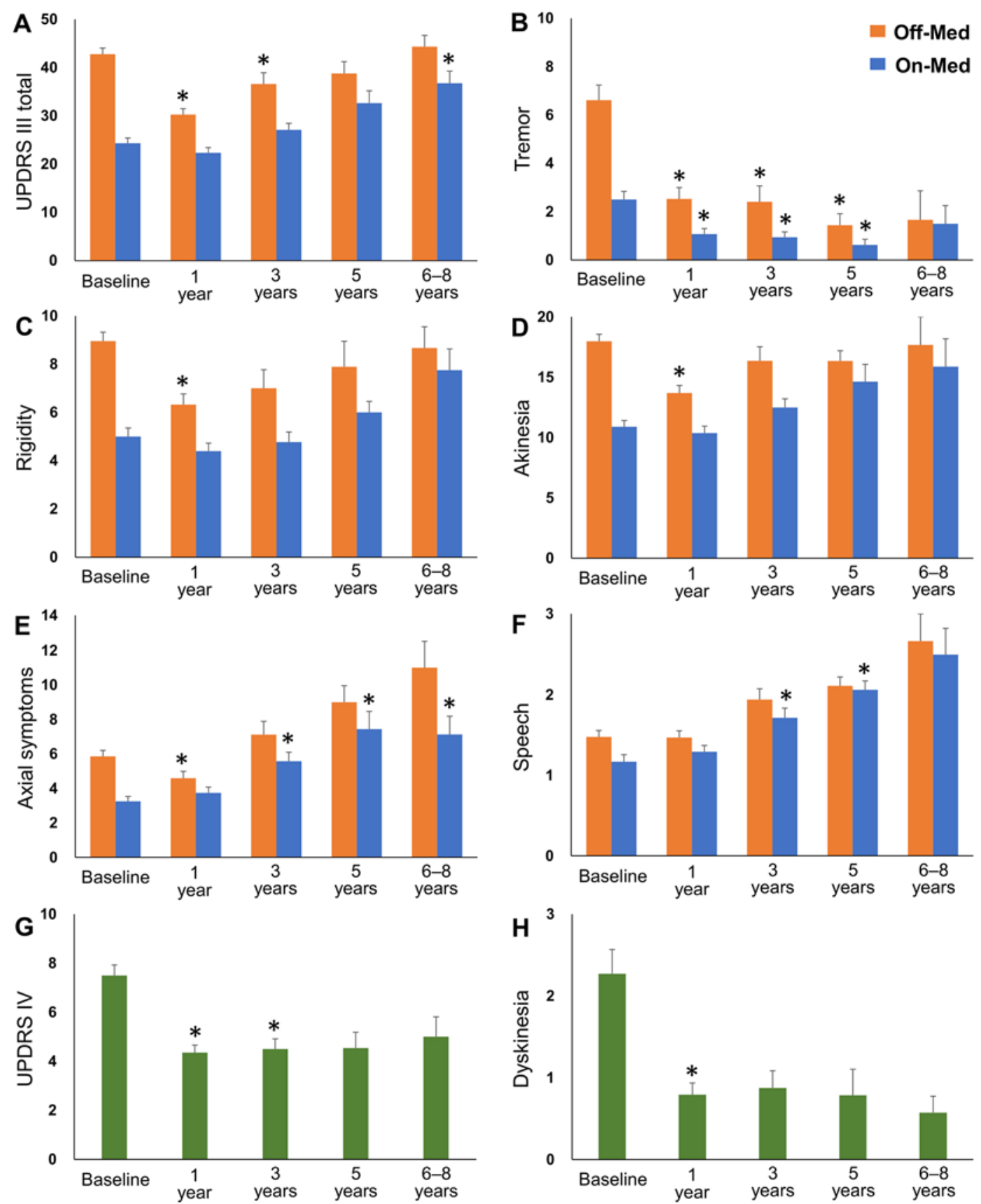

FIG. 1. Longitudinal changes of UPDRS III total (A), tremor total (items 20 and 21, B), rigidity (item 22, C), akinesia total (D), axial symptoms total (items 23-26 and 31, E), speech (item 18, F), UPDRS IV (G), and dyskinesia total (items 32-34, H) scores are shown. Orange and blue bars in A-F represent the mean scores for the off- and on-medication states, respectively. Whiskers represent the standard error. An asterisk indicates significant differences between the baseline and postoperative scores. Med = medication. Figure is available in color online only.

and stimulation-induced double vision $(\mathrm{n}=1)$. Most stimulation-related AEs were mild and managed by adjusting programming settings.

\section{Discussion}

In this single-center retrospective study, we aimed to investigate the long-term outcomes of bilateral GPi DBS for patients with PD for 5 years and longer. In our cohort, the patients experienced significant improvement in the off-medication UPDRS III total scores by 12.5 points at 1 year after GPi implantation as compared with baseline, which was comparable to the findings from the large DBS trials (11.4- to 11.7-point improvement at 6 months or 1 year) ${ }^{2,15}$ The benefits for tremor, rigidity, akinesia, gait, postural stability, motor complications, and dyskinesia in the short term were also similar to those in the earlier studies., ${ }^{4,615-21}$ However, the off-medication UPDRS III 
TABLE 4. Percentage improvement of UPDRS IV and dyskinesia scores in individual patients

\begin{tabular}{|c|c|c|c|c|c|c|c|c|}
\hline \multirow[b]{2}{*}{ Variable } & \multicolumn{4}{|c|}{ UPDRS IV } & \multicolumn{4}{|c|}{ Dyskinesia } \\
\hline & $1 Y r$ & 3 Yrs & 5 Yrs & $6-8$ Yrs & $1 \mathrm{Yr}$ & 3 Yrs & 5 Yrs & $6-8$ Yrs \\
\hline No. of patients & 49 & 28 & 12 & 7 & 39 & 23 & 10 & 5 \\
\hline \multicolumn{9}{|l|}{ Improvement } \\
\hline$>50 \%$ & $22(45 \%)$ & $10(36 \%)$ & $4(33 \%)$ & $2(29 \%)$ & $32(82 \%)$ & $15(65 \%)$ & $9(90 \%)$ & $4(80 \%)$ \\
\hline $30 \%-50 \%$ & $8(16 \%)$ & $2(7 \%)$ & $1(8 \%)$ & $1(14 \%)$ & $1(3 \%)$ & $1(4 \%)$ & $0(0 \%)$ & $0(0 \%)$ \\
\hline$<30 \%$ & $19(39 \%)$ & $16(57 \%)$ & $7(58 \%)$ & $4(57 \%)$ & $6(15 \%)$ & $6(26 \%)$ & $1(10 \%)$ & $1(20 \%)$ \\
\hline
\end{tabular}

Percentage improvement from baseline scores is presented as the number of patients (\%).

total scores declined over time with nonsignificant improvement at 5 years compared with baseline scores. The on-medication UPDRS III total scores also worsened over time, along with rigidity, akinesia, speech, gait, and postural stability. In contrast, tremor total scores in both the off- and on-medication states, UPDRS IV, and dyskinesia scores showed similar magnitudes of improvement at the short- and long-term assessments (the ranges of the percentage improvement at each time point: $61 \%-75 \%, 30 \%-$ $80 \%, 29 \%-40 \%$, and $40 \%-65 \%$, respectively) despite not meeting statistical significance at 5 years or later, most likely due to diminishing sample size. The significant initial improvement in the ADL and discomfort domains of the PDQ-39 was lost in the long term, and there was a gradual worsening in all domains.

To the best of our knowledge, long-term outcomes of GPi DBS for 5 years and beyond have been available for only 30 PD patients across 3 studies. ${ }^{4-6}$ The results from these 3 studies are summarized in Table 7, and they are shown with the data from the present study. The percentage improvement of the off-medication UPDRS III at 5 years ranged between $7 \%$ and $35 \%$, and only the study by Moro et al. showed significant improvement in the offmedication UPDRS III at 5 years when compared with baseline. ${ }^{5}$ The percentage improvement of the on-medication UPDRS III at 5 years ranged between $-28 \%$ and $16 \%$. We observed significant worsening in the on-medication UPDRS III at 6-8 years, which was consistent with the results from Lachenmayer et al.'s study. ${ }^{6}$ The worsening of the on-medication UPDRS III over time suggests that disease progression likely played the major role, although we did not assess the impact of disease progression through a washout (e.g., by switching off the stimulation).

The present study investigated the effect of GPi DBS on tremor at 5 years or longer follow-up. There have been concerns of limited efficacy and tremor recurrence over time with the GPi target. GPi DBS, however, provided marked sustained resting and postural/action tremor improvement in both the off- and on-medication states for 5 years of follow-up. The two earlier long-term GPi DBS studies also reported significant improvement in the offmedication tremor scores at 5 years, whereas the present study is the first one that reports significant improvement in the on-medication tremor scores at 5 years. ${ }^{4-6}$ Furthermore, our findings are in agreement with a recent metaanalysis of large randomized trials with follow-up periods of 1-3 years, in which DBS suppressed tremor regardless of the medication state. ${ }^{22}$ Importantly, the benefits in tremor were comparable between GPi and STN DBS in this meta-analysis. Therefore, GPi and STN DBS are likely equally effective for tremor control in both the onand off-medication states, although long-term comparative data between these targets beyond 3 years remain scarce. ${ }^{5}$

Long-term GPi studies have reported variable outcomes in rigidity control in the off-medication state, ${ }^{4-6}$ and we did not observe significant benefits at 5 years and later. Similarly, significant improvement in akinesia and axial symptoms in the off-medication state was lost by 3 years. These findings concur with those of the earlier studies that reported nonsignificant improvement in akinesia and axial symptoms at 5 years. ${ }^{4-6}$ In contrast, UPDRS IV and dyskinesia scores showed similar magnitudes of improvement at the short- and long-term assessments (the ranges of the percentage improvement at each time point were $29 \%$ $40 \%$ and $40 \%-65 \%$, respectively) despite not meeting statistical significance at 5 years or later. We suspect that the lack of statistical power underpinned the failure to reach significance. The earlier GPi studies consistently showed significant improvement of dyskinesia at 5 years. ${ }^{4-6}$

HRQoL is an important outcome measure to assess

TABLE 5. Percentage improvement of tremor scores in individual patients

\begin{tabular}{|c|c|c|c|c|c|c|c|c|}
\hline \multirow[b]{2}{*}{ Variable } & \multicolumn{4}{|c|}{ Off-Med Tremor Total Scores } & \multicolumn{4}{|c|}{ On-Med Tremor Total Scores } \\
\hline & $1 \mathrm{Yr}$ & 3 Yrs & $5 \mathrm{Yrs}$ & $6-8$ Yrs & $1 \mathrm{Yr}$ & 3 Yrs & 5 Yrs & $6-8$ Yrs \\
\hline No. of patients & 48 & 17 & 9 & 3 & 49 & 29 & 12 & 5 \\
\hline \multicolumn{9}{|l|}{ Improvement } \\
\hline$>50 \%$ & $35(73 \%)$ & $12(71 \%)$ & $7(78 \%)$ & $2(67 \%)$ & $32(65 \%)$ & $21(72 \%)$ & $9(75 \%)$ & $2(40 \%)$ \\
\hline $30 \%-50 \%$ & $5(10 \%)$ & $1(6 \%)$ & $0(0 \%)$ & $0(0 \%)$ & $6(12 \%)$ & $1(3 \%)$ & $1(8 \%)$ & $0(0 \%)$ \\
\hline$<30 \%$ & $8(17 \%)$ & $4(24 \%)$ & $2(22 \%)$ & $1(33 \%)$ & $11(22 \%)$ & $7(24 \%)$ & $2(17 \%)$ & $3(60 \%)$ \\
\hline
\end{tabular}

Percentage improvement from baseline scores is presented as the number of patients (\%). 
TABLE 6. DBS programming parameters

\begin{tabular}{lcccc}
\hline \multicolumn{1}{c}{ Parameter } & 1 Yr & 3 Yrs & 5 Yrs & $6-8$ Yrs \\
\hline Voltage $(\mathrm{V})$ & $2.8(1.6-4.6)$ & $2.7(0.8-3.8)$ & $3.0(1.6-3.8)$ & $3.0(1.6-3.7)$ \\
\hline Pulse width $(\mu \mathrm{sec})$ & $90(60-150)$ & $90(75-150)$ & $90(75-135)$ & $90(75-135)$ \\
\hline Frequency $(\mathrm{Hz})$ & $160(135-200)$ & $160(120-200)$ & $150(120-185)$ & $150(120-175)$ \\
\hline \multicolumn{7}{l}{ Data are presented as median (range). }
\end{tabular}

the efficacy of any intervention. Based on the PDQ-39, we found significant improvement in the ADL and bodily discomfort domains at 1 year. However, this benefit was lost at 3 years and was followed by worsening over time in all the domains. In the large GPi DBS trials where the PDQ-39 was used for HRQoL assessments, the investigators reported a significant improvement in different domains, including the mobility, ADL, emotional well-being, stigma, cognition, communication, and bodily discomfort domains, with follow-up periods ranging from 8 months to 3 years.,23 The inconsistent findings across studies may be related to the differences in motor and nonmotor profiles and baseline PDQ-39 in the study populations. A series of studies have reported on the effectiveness and safety of GPi DBS in PD patients with a contraindication for STN DBS inclusive of cognitive dysfunction or dopa-resistant axial symptoms. ${ }^{19,23}$ Significant improvement in motor function and dyskinesia was maintained for 3 years with a reasonable neuropsychological safety profile..$^{23}$ However, there was no significant improvement in HRQoL. These results suggest that nonmotor symptoms and axial symptoms are possibly associated with PD GPi DBS outcomes. Future studies should investigate nonmotor outcomes of GPi DBS. ${ }^{1}$

Thus far, randomized controlled trials comparing GPi and STN DBS have shown comparable benefits for motor symptoms, dyskinesia, and HRQoL between these targets for 3 years following DBS implantation., ${ }^{2,3}$ A significant reduction in antiparkinsonian medications after STN DBS can be achieved in most cases. Some authors have suggested that STN DBS may improve some adverse effects of medications, such as sedation, dyskinesia, or hyperdopaminergic behavioral disorders, but that the medication reduction may exacerbate apathy and neuropsychiatric problems. ${ }^{1,24}$ In contrast, GPi DBS may provide better out- comes for dyskinesia and potentially cognitive function and axial motor function as compared with STN DBS. ${ }^{1}$ A recent extensive review summarized 5-year outcomes of STN DBS for 551 PD patients from 15 studies. ${ }^{24}$ In most studies, substantial improvement in tremor and rigidity in the off-medication state and dyskinesia were maintained at 5 years and beyond, while benefits in akinesia and axial symptoms decreased between the 1- and 5-year assessments. In the on-medication state, worsening of motor symptoms, especially in akinesia and axial symptoms, was also observed. Likewise, 4 studies, which used the PDQ39 for HRQoL assessments, consistently reported that the benefits seen at 1 year were primarily lost by 5 years. ${ }^{25-28}$ These long-term outcomes of STN DBS seem to be comparable to those of the present study and other GPi DBS studies, but it is difficult to make such a direct comparison because of the considerable differences in the clinical profiles and study designs in earlier studies. More long-term motor, nonmotor, and HRQoL outcomes from randomized controlled trials will be needed to establish a tailored DBS treatment strategy for individual patients. Additionally, large DBS studies should be compared on baseline disease characteristics, especially levodopa response, as was recently reported by Deuschl et al..$^{29}$

In the present study, medication changes and AEs following DBS implantation and DBS programming parameters were comparable to those from earlier reports. In our cohort, there were no significant changes in LEDD postoperatively. Past studies have similarly reported stable medication dosages following GPi DBS. ${ }^{4-6}$ The most frequent AEs included gait/postural disorders, dysarthria, and lead breakage, which were also reported as frequent AEs in large clinical DBS studies for PD patients. ${ }^{3,16}$ GPi DBS programming parameters revealed wider pulse widths and higher frequencies when compared with STN DBS., ${ }^{3,46}$

TABLE 7. Studies reporting 5-year outcomes of GPi DBS in patients with PD

\begin{tabular}{|c|c|c|c|c|c|c|c|}
\hline \multirow{2}{*}{$\begin{array}{l}\text { Authors \& } \\
\quad \text { Year }\end{array}$} & \multirow{2}{*}{$\begin{array}{l}\text { Total No. } \\
\text { of Patients } \\
\text { Analyzed }\end{array}$} & \multirow{2}{*}{$\begin{array}{l}\text { No. of } \\
\text { Patients } \\
\text { at } 5 \text { Yrs }\end{array}$} & \multirow{2}{*}{$\begin{array}{l}\text { Mean Age } \\
\text { at DBS } \\
\text { (SD), Yrs }\end{array}$} & \multirow{2}{*}{$\begin{array}{l}\text { Mean Disease } \\
\text { Duration Before } \\
\text { DBS (SD), Yrs }\end{array}$} & \multicolumn{2}{|c|}{$\begin{array}{c}\text { \% Improvement of UPDRS III } \\
\text { at } 5 \text { Yrs From BL }\end{array}$} & \multirow{2}{*}{$\begin{array}{l}\text { Items That Maintained } \\
\text { Significant Improvement at } 5 \text { Yrs } \\
\text { Compared w/ BL }\end{array}$} \\
\hline & & & & & Off-Med & On-Med & \\
\hline $\begin{array}{l}\text { Volkmann et } \\
\text { al., } 2004^{4}\end{array}$ & 11 & 6 & $45.8(8.6)$ & $11.2(2.7)$ & $23 \%$ & $16 \%$ & Off-med rigidity score, dyskinesia score \\
\hline $\begin{array}{l}\text { Moro et al., } \\
2010^{5}\end{array}$ & 16 & 16 & $56.0(8.2)$ & $14.0(5.2)$ & $35 \% *$ & $-17 \%$ & $\begin{array}{l}\text { Off-med rigidity \& tremor scores, off-med } \\
\text { ADL score, dyskinesia score }\end{array}$ \\
\hline $\begin{array}{l}\text { Lachenmayer } \\
\text { et al., } 2019^{6}\end{array}$ & 18 & 8 & $64.8(7.4)$ & $16.2(6.8)$ & $7 \%$ & $-28 \%{ }^{*}$ & Off-med tremor score, dyskinesia score \\
\hline Present study & 65 & 16 & $62.6(10.5)$ & $11.9(5.4)$ & $20 \%$ & $-14 \% *$ & Off-med tremor score, on-med tremor score \\
\hline
\end{tabular}

${ }^{*}$ Reported statistical significances. 
We would like to highlight the limitations of our study. First, this is a single-center retrospective study, and all assessments were open-label without a control group. Second, off-DBS assessments were not performed, and therefore we could not determine the impact of disease progression quantitively. Third, 18\% (12 of 65) of the patients failed to complete the visits. We could not exclude the possibility that the patients with worse DBS outcomes or faster disease progression might have dropped out more frequently. Although the clinical backgrounds were comparable between the patients who completed regular visits and those who did not, the dropouts could have affected long-term outcomes. Fourth, we could not analyze postoperative changes in the neuropsychological data because of insufficient data. Fifth, the relatively small number of patients for the long-term assessments limited the statistical power. Sixth, the selection of GPi over STN DBS at the interdisciplinary DBS discussion likely biased this cohort to be those with more severe PD and those with dyskinesia. Finally, we did not assess the nonmotor symptoms in these patients. The retrospective nature of the present study impeded detailed assessments of DBS programming parameters in individual patients.

\section{Conclusions}

The present study reported long-term outcomes of bilateral GPi DBS for PD patients for 5 years and longer. Significant improvement in the off-medication UPDRS III total scores was lost at 5 years following GPi implantation. The off- and on-tremor scores, UPDRS IV, and dyskinesia scores showed stable improvement over time, whereas the benefits for rigidity, akinesia, gait, and postural stability in the off-medication state declined. The worsening of motor scores in the on-medication state was possibly related to disease progression. Similarly, initial benefits in the ADL and discomfort domains of the PDQ-39 were lost in the long term, along with a gradual worsening in all the HRQoL domains. In future studies, preoperative clinical subtypes, disease progression, and long-term outcomes, as well as the effects on nonmotor symptoms, should be further explored. Repeating on- and off-medication testing each year could shine even more light on the disease progression issue.

\section{References}

1. Ramirez-Zamora A, Ostrem JL. Globus pallidus interna or subthalamic nucleus deep brain stimulation for Parkinson disease: a review. JAMA Neurol. 2018;75(3):367-372.

2. Weaver FM, Follett KA, Stern M, et al. Randomized trial of deep brain stimulation for Parkinson disease: thirty-sixmonth outcomes. Neurology. 2012;79(1):55-65.

3. Odekerken VJ, Boel JA, Schmand BA, et al. GPi vs STN deep brain stimulation for Parkinson disease: Three-year follow-up. Neurology. 2016;86(8):755-761.

4. Volkmann J, Allert N, Voges J, et al. Long-term results of bilateral pallidal stimulation in Parkinson's disease. Ann Neurol. 2004;55(6):871-875.

5. Moro E, Lozano AM, Pollak P, et al. Long-term results of a multicenter study on subthalamic and pallidal stimulation in Parkinson's disease. Mov Disord. 2010;25(5):578-586.

6. Lachenmayer ML, Bettschen C, Bernasconi C, et al. Stimulation of the globus pallidus internus in the treatment of Par- kinson's disease: long-term results of a monocentric cohort. Parkinsonism Relat Disord. 2019;64:118-123.

7. Hughes AJ, Daniel SE, Kilford L, Lees AJ. Accuracy of clinical diagnosis of idiopathic Parkinson's disease: a clinicopathological study of 100 cases. J Neurol Neurosurg Psychiatry. 1992;55(3):181-184.

8. Tsuboi T, Jabarkheel Z, Foote KD, et al. Importance of the initial response to GPi deep brain stimulation in dystonia: a nine year quality of life study. Parkinsonism Relat Disord. 2019;64:249-255.

9. Fahn S, Elton RL. Unified Parkinson's disease rating scale. IN: Fahn S, Marsden CD, Goldstein M, Calne DB, eds. Recent Developments in Parkinson's Disease. Macmillan; 1987: $153-163$.

10. Tomlinson CL, Stowe R, Patel S, et al. Systematic review of levodopa dose equivalency reporting in Parkinson's disease. Mov Disord. 2010;25(15):2649-2653.

11. Mattis S. Dementia Rating Scale-2: Professional Manual. Psychological Assessment Resources; 2001.

12. Beck A, Steer R, Brown G. Manual for Beck Depression Inventory-II. Psychological Corporation; 1996.

13. Starkstein SE, Jorge R, Mizrahi R. The prevalence, clinical correlates and treatment of apathy in Alzheimer's disease. Eur J Psychiatry. 2006;20(2):96-106.

14. Holm S. A simple sequentially rejective multiple test procedure. Scand J Stat. 1979;6(2):65-70.

15. Odekerken VJ, van Laar T, Staal MJ, et al. Subthalamic nucleus versus globus pallidus bilateral deep brain stimulation for advanced Parkinson's disease (NSTAPS study): a randomised controlled trial. Lancet Neurol. 2013;12(1):37-44.

16. Follett KA, Weaver FM, Stern M, et al. Pallidal versus subthalamic deep-brain stimulation for Parkinson's disease. $N$ Engl J Med. 2010;362(22):2077-2091.

17. Obeso JA, Olanow CW, Rodriguez-Oroz MC, et al. Deepbrain stimulation of the subthalamic nucleus or the pars interna of the globus pallidus in Parkinson's disease. $N$ Engl $J$ Med. 2001;345(13):956-963.

18. Anderson VC, Burchiel KJ, Hogarth P, et al. Pallidal vs subthalamic nucleus deep brain stimulation in Parkinson disease. Arch Neurol. 2005;62(4):554-560.

19. Rouaud T, Dondaine T, Drapier S, et al. Pallidal stimulation in advanced Parkinson's patients with contraindications for subthalamic stimulation. Mov Disord. 2010;25(12):1839_ 1846.

20. Volkmann J, Allert N, Voges J, et al. Safety and efficacy of pallidal or subthalamic nucleus stimulation in advanced PD. Neurology. 2001;56(4):548-551.

21. Tsuboi T, Lemos Melo Lobo Jofili Lopes J, Patel B, et al. Parkinson's disease motor subtypes and bilateral GPi deep brain stimulation: one-year outcomes. Parkinsonism Relat Disord. 2020;75:7-13.

22. Wong JK, Cauraugh JH, Ho KWD, et al. STN vs. GPi deep brain stimulation for tremor suppression in Parkinson disease: a systematic review and meta-analysis. Parkinsonism Relat Disord. 2019;58:56-62.

23. Bonenfant J, Drapier S, Houvenaghel JF, et al. Pallidal stimulation in Parkinson's patients with contraindications to subthalamic target: a 3 years follow-up. Parkinsonism Relat Disord. 2017;34:20-25.

24. Limousin P, Foltynie T. Long-term outcomes of deep brain stimulation in Parkinson disease. Nat Rev Neurol. 2019;15(4): 234-242.

25. Siderowf A, Jaggi JL, Xie SX, et al. Long-term effects of bilateral subthalamic nucleus stimulation on health-related quality of life in advanced Parkinson's disease. Mov Disord. 2006;21(6):746-753.

26. Aviles-Olmos I, Kefalopoulou Z, Tripoliti E, et al. Long-term outcome of subthalamic nucleus deep brain stimulation for Parkinson's disease using an MRI-guided and MRI-verified 
approach. J Neurol Neurosurg Psychiatry. 2014;85(12):14191425.

27. Jiang LL, Liu JL, Fu XL, et al. Long-term efficacy of subthalamic nucleus deep brain stimulation in Parkinson's disease: a 5-year follow-up study in China. Chin Med J (Engl). 2015; 128(18):2433-2438.

28. Lezcano E, Gómez-Esteban JC, Tijero B, et al. Long-term impact on quality of life of subthalamic nucleus stimulation in Parkinson's disease. J Neurol. 2016;263(5):895-905.

29. Deuschl G, Follett KA, Luo P, et al. Comparing two randomized deep brain stimulation trials for Parkinson's disease. $J$ Neurosurg. 2020;132(5):1376-1384.

\section{Disclosures}

D.B. has received research grants from the Parkinson's Foundation, NIH, and the McKnight Research Foundation. L.A. works as a consultant and participates in advisory boards for Boston Scientific and Medtronic and has received honoraria for these services. K.D.F. reports grants from NIH and other funding from the Donnellan/Einstein/Merz Chair (no direct remuneration to K.D.F.) during this study; grants and nonfinancial support from Medtronic; grants from St. Jude, Functional Neuromodulation, and Boston Scientific; and grants and other funding from NeuroPace. Additionally, K.D.F. has a patent (US 8295935 B2) issued for a DBS cranial lead fixation device that was not used for this research and for which no personal remuneration or royalty has been realized for K.D.F. M.S.O. serves as a consultant for the Parkinson's Foundation and has received research grants from NIH, the Parkinson's Foundation, the Michael J. Fox Foundation, the Parkinson Alliance, the Smallwood Foundation, the BachmannStrauss Foundation, the Tourette Syndrome Association, and the UF Foundation. M.S.O. has received royalties for publications with Demos, Manson, Amazon, Smashwords, Books4Patients, Perseus, Robert Rose, Oxford, and Cambridge (movement disorders books). M.S.O. is an associate editor for New England Journal of Medicine and Journal Watch Neurology, and is also an associate editor for JAMA Neurology. M.S.O. has participated in CME and educational activities on movement disorders sponsored by the Academy for Healthcare Learning, PeerView, Prime, QuantiaMD, WebMD/Medscape, Medicus, MedNet, Einstein,
Henry Stewart, the American Academy of Neurology, the Movement Disorders Society, and Vanderbilt University. The institution, not M.S.O., receives grants from Medtronic, Abbvie, Boston Scientific, Abbott, and Allergan, and the PI has no financial interest in these grants. M.S.O. has participated as a site PI and/or coinvestigator for several $\mathrm{NIH}$, foundation, and industry-sponsored trials over the years but has not received honoraria. Research projects at the University of Florida receive device and drug donations. A.R.Z. serves as a consultant for the National Parkinson Foundation and has received research consulting honoraria from Medtronic, Boston Scientific, CNS Ratings, and Bracket.

\section{Author Contributions}

Conception and design: Tsuboi, Legacy, Okun, Ramirez-Zamora. Acquisition of data: Lemos Melo Lobo Jofili Lopes, Moore, Patel, Ratajska, Bowers, Eisinger, Almeida, Foote. Analysis and interpretation of data: Tsuboi, Ratajska, Bowers, Ramirez-Zamora. Drafting the article: Tsuboi. Critically revising the article: Lemos Melo Lobo Jofili Lopes, Moore, Patel, Legacy, Eisinger, Almeida, Foote, Okun, Ramirez-Zamora. Reviewed submitted version of manuscript: Tsuboi, Lemos Melo Lobo Jofili Lopes, Moore, Patel, Legacy, Eisinger, Almeida, Foote, Okun, Ramirez-Zamora. Approved the final version of the manuscript on behalf of all authors: Tsuboi. Statistical analysis: Tsuboi. Study supervision: Okun, Ramirez-Zamora.

\section{Supplemental Information}

Online-Only Content

Supplemental material is available with the online version of the article.

Supplementary Tables 1-3. https://thejns.org/doi/suppl/10. 3171/2020.6.JNS20617.

\section{Correspondence}

Takashi Tsuboi: Norman Fixel Institute for Neurological Diseases, Gainesville, FL.takashi80@gmail.com; takashi.tsuboi@ neurology.ufl.edu. 